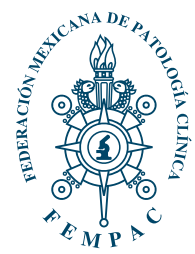

\title{
Secuenciación de la CVP3A4, evaluación de la actividad de la colinesterasa y geolocalización de trabajadores expuestos en comunidad agraria de la Colonia Tovar, Venezuela
}

\section{Palabras clave: \\ Plaguicidas \\ organofosforados, \\ carbamatos, \\ colinesterasa, \\ salud ocupacional, \\ geolocalización. \\ Keywords: \\ Organophosphorus \\ pesticides, carbamates, \\ cholinesterase, \\ occupational health, geolocation.}

* Unidad de Toxicología Molecular (UTM).

Escuela de Bioanálisis.

Facultad de Ciencias de

la Salud. Universidad de

Carabobo. Laboratorio

de Referencia

Marrero Blanco.

Venezuela. Doctorado

Individualizado,

Facultad de Medicina,

Universidad Central

de Venezuela.

Caracas, Venezuela.

¥ Departamento

de Microbiología

de la Escuela de

Ciencias Biomédicas

y Tecnológicas de

la Universidad de

Carabobo, Venezuela.

Recibido:

05/05/2020

Aceptado:

$16 / 06 / 2020$

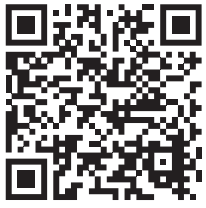

Sequencing of CYP3A4 and evaluation of the activity of the colinesterase and geolocalization of workers exposed in the agrarian community of Colonia Tovar, Venezuela

Marrero Sharim,* Sequera Mónica ${ }^{\ddagger}$

RESUMEN

El cambio climático genera fenómenos extremos, lo que conlleva a la reducción de la producción agrícola y al aumento del uso de plaguicidas (organofosforados y carbamatos) causando una inhibición de la acetilcolinesterasa. Este estudio evaluó la exposición a estos plaguicidas en 20 trabajadores agrícolas del caserío Capachal de Palmarito, Colonia Tovar determinando la actividad de colinesterasa plasmática, expresión de CYP3A4 y el uso de plaguicidas en el ambiente. Se obtuvo la media de la actividad de la colinesterasa plasmática dentro de los parámetros normales $(4,970-13,977 \mathrm{U} / \mathrm{L})$ en grupos expuesto y control; tres trabajadores expuestos se ubicaron por debajo del rango de normalidad, siendo esto estadísticamente significativo $(p=0.005)$. Las variantes alélicas encontradas para CYP3A4 no presentaron relación con la exposición o efecto del xenobiótico en el genotipo de los individuos estudiados. En la geolocalización se detectó el predominio de sujetos con baja actividad colinesterásica en el cuadrante suroeste del caserío agrícola, lo que sugiere que esta área tiene una actividad agrícola intensa y por ende mayor uso de plaguicidas.

\section{ABSTRACT}

Climate change generates extreme phenomena, leading to reduced agricultural production, increasing the use of pesticides (organophosphates and carbamates) causing an inhibition of acetylcholinesterase. This study evaluated the exposure to these pesticides in 20 agricultural workers from the Capachal de Palmarito farm, Colonia Tovar; determining the activity of plasma cholinesterase, CYP3A4 expression and the use of pesticides in the environment. The mean of the cholinesterase activity was obtained within the normal parameters (4,97013,977 U/L) in exposed and control groups; three workers are below the normal range, being statistically significant $(p=0.005)$. The allelic variants found for CYP3A4 have no relationship with the exposure or effect of the xenobiotic on the genotype of the individuals studied. In geolocation, the predominance of subjects with low cholinesterase activity is detected in the south-west quadrant of the agricultural hamlet, suggesting that this area has intense agricultural activity and greater use of pesticides.

\section{INTRODUCCIÓN}

A medida que las sociedades se desarrollan, aumentan su densidad poblacional, tienen tecnologías más complejas y por supuesto mayor necesidad de rentabilizar al máximo posible

Correspondencia:

Prof. Sharim Marrero

Tel: + 584166482689

E-mail: smarrero@uc.edu.ve / sharimmarrerob@hotmail.com la tierra, lo que ha propiciado el intenso uso de plaguicidas. ${ }^{1}$

Miles de toneladas de plaguicidas son introducidas cada año en el medio ambiente con la intención de controlar una amplia variedad de fenómenos relacionados con las prácticas agrícolas. ${ }^{2}$ Es por ello que la Organización para la Alimentación y la Agricultura (FAO), durante la Conferencia de las Naciones Unidas sobre el Cambio Climático (COP23) en 2017, indicó 
que la población mundial crecerá en 2,000 millones de habitantes para el año 2050, por lo que la producción agrícola tendrá que aumentar $60 \%$ para poder mitigar la pobreza y adaptarse al cambio climático y por ende el incremento en el uso de plaguicidas será mayor. ${ }^{3,4}$

El cambio climático siguió siendo el tema relevante en la COP25 del año 2019, evidenciándose que los acuerdos y planes no están respondiendo de forma efectiva a la crisis climática global, al impacto de la pérdida de diversidad y a los riesgos que enfrenta la tierra, los océanos y la criósfera que repercuten en la producción de alimentos, ${ }^{5}$ ya que genera mayor frecuencia de fenómenos extremos y ausencia de predictibilidad de los patrones meteorológicos.

Los plaguicidas son los responsables de un elevado número de casos de intoxicación cuando el producto está clasificado como de toxicidad aguda, mientras que los productos con efectos tóxicos crónicos pueden provocar cáncer o trastornos hasta en la población no expuesta ocupacionalmente. Es por ello que la FAO y la Organización Mundial para la Salud (OMS) pretenden reducir los daños causados por los plaguicidas, los cuales representan un riesgo tóxico para la salud y el medio ambiente, ${ }^{6,7}$ basándose en los estudios del Centro Internacional de Investigaciones sobre el Cáncer (CIIC) que relacionan de manera constante el uso de plaguicidas con efectos a largo plazo. ${ }^{8,9}$

Durante años se ha promovido la venta de plaguicidas sin informar adecuadamente el riesgo inherente a su uso, además algunos de ellos son considerados compuestos orgánicos persistentes (COP), es por ello que el Convenio de Estocolmo ${ }^{10}$ fue el primer gran paso necesario para empezar a mitigar, vigilar, controlar, analizar, los efectos de los COP y eliminar sus fuentes. A este convenio le siguieron otros también internacionales como el de Rotterdam, Basilea y por último y más reciente el Código de Conducta para la Distribución y Uso de Plaguicidas, todos ellos permiten generar en cada país y entre países, planes, proyectos, normas y procedimientos que regulan el uso, transporte y eliminación de sustancias químicas y desechos peligrosos. ${ }^{7,10}$

La FAO en su informe 2015-2030 indica que «el uso de plaguicidas se ha incrementado considerablemente a lo largo de los últimos 35 años, alcanzando tasas de crecimiento del 4 al $5.4 \%$ en algunas regiones». En el futuro se espera el uso de plaguicidas «inteligentes», variedades de cultivos resistentes y métodos ecológicos de control de plagas, llamados enfoques de manejo integrado de plagas (MIP) tengan por objetivo final reducir la dependencia de plaguicidas. ${ }^{11}$

Dentro de la producción agrícola, el número de trabajadores activos a nivel mundial es de 1,300 millones, lo cual corresponde al 50\% de la mano de obra en el mundo. Según las estimaciones de la Oficina Internacional del Trabajo (OIT), un mínimo de 170.000 trabajadores agrícolas muere cada año, siendo víctimas de lesiones graves causadas por accidentes con maquinaria o por envenenamiento con plaguicidas $u$ otros agroquímicos. ${ }^{12}$

Los países en vías de desarrollo, a pesar de algunos esfuerzos en positivo, todavía tienen deficiencias importantes en algunos aspectos del manejo de los plaguicidas, entre estos países destaca Venezuela, aun cuando firmó el Convenio de Estocolmo el 23 de mayo de 2001 y lo ratificó el 03 de enero de 2005, según Gaceta Oficial 38.098, «lo que implica su incorporación a la legislación como Ley Aprobatoria o Ley Supra en el artículo 23 de la Constitución de la República Bolivariana de Venezuela» ${ }^{11,13}$ y en los artículos 127 y 129 del Capítulo IX de los Derechos Ambientales, Ley № 55 sobre sustancias, materiales y desechos peligrosos en su artículo 7, además de la Norma COVENIN 2709 de 2002. ${ }^{14}$

En cuanto a los eventos de notificación obligatoria en Venezuela, según el boletín epidemiológico de la semana 32 de 2014 que señala entre las principales causas de consulta las intoxicaciones por plaguicidas en $0.01 \%$, existen además 463 casos de intoxicaciones por plaguicidas acumulados hasta el mes de agosto del respectivo año. ${ }^{15}$ Cabe destacar que la Federación de Fabricantes de Productos Químicos Agropecuarios AFAQUIMA da seguimiento a los casos de intoxicaciones por el uso de plaguicidas y otros químicos utilizados en las actividades agrícolas, reportando cualquier cambio. ${ }^{16}$

Los principales plaguicidas utilizados hoy día pertenecen al grupo de los carbamatos, organofosforados (OF), tiocarbamatos y piretroides. Los OF y carbamatos causan una inhibición de la acetilcolinesterasa (enzima encargada de degradar la acetilcolina en el espacio sináptico), lo que conduce a una acumulación del neurotransmisor acetilcolina en los receptores muscarínicos y nicotínicos con la consiguiente hiperestimulación del sistema parasimpático, dando como resultado un síndrome colinérgico. ${ }^{17}$

El principal responsable del metabolismo oxidativo de los xenobióticos es la citocromo P-450, específicamente la correspondiente a la subfamilia con interés fármacotoxicológico CYP3A4, que incluye enzimas inducibles por hidrocarburos aromáticos (plaguicidas, herbicidas, solventes) que participan en la fase I del metabolismo de xenobióticos. ${ }^{18}$ Estos últimos se incluyen dentro de los llamados biomarcadores de susceptibilidad, los cuales se basan en identificar las diferencias interindividuales que hacen que un individuo sea más susceptible o responda de manera diferente, con mayor riesgo para su salud, frente a diferentes exposiciones ambientales. 
Las diferencias entre individuos en la expresión o actividad de las enzimas CYP se deben a factores fisiológicos, patológicos, ambientales y/o genéticos. Estos últimos pueden corresponder a repeticiones nucleotídicas, inserciones, deleciones o polimorfismos de un nucleótido (SNPs), lo que modifica la secuencia aminoacídica de las proteínas codificadas y la expresión génica. ${ }^{19}$

En el presente estudio se evaluó la exposición a OF y carbamatos en trabajadores agrícolas del caserío Capachal de Palmarito de la Colonia Tovar mediante la determinación de la actividad de la colinesterasa plasmática, signos y síntomas y el uso y manejo de plaguicidas. Asimismo, se midieron parámetros hematológicos, bioquímicos de funcionalismo renal y hepático, la geolocalización de los trabajadores, además de la detección de polimorfismo de la isoenzima CYP3A4.

\section{MATERIAL Y MÉTODOS}

Se realizó un estudio descriptivo-correlacional para identificar la prevalencia de sintomatología persistente asociada con exposición a plaguicidas y su relación con los niveles de colinesterasa sanguínea, parámetros hematológicos, bioquímicos de funcionalismo renal y hepático y la expresión de la CYP3A4. El estudio se llevó a cabo entre los meses de junio y octubre de 2017 en la Colonia Tovar, Estado Aragua, Venezuela, a unos 63 $\mathrm{km}$ al oeste de la ciudad de Caracas con una población censada por el Consejo Comunal de 57 agricultores. La muestra estuvo representada por un total de 20 trabajadores ocupacionalmente expuestos; mientras que el grupo control estuvo constituido por 10 sujetos del personal que labora en el área administrativa comercial de la ciudad de Valencia, Estado Carabobo-Venezuela, a $160 \mathrm{~km}$ aproximadamente al oeste de Caracas. En los dos grupos se trabajó con personas de ambos géneros, con edades comprendidas entre 21 y 58 años que aceptaron participar en el estudio. El criterio de inclusión para la selección de la muestra fue: ser trabajador agrícola expuesto por un periodo no menor de seis meses, con aparente buena condición de salud, sin hábitos psicosociales (fumar, ingesta de alcohol) y respecto al grupo control se tomó en cuenta la zona residencial y actividad laboral del sujeto, alejado de contacto con plaguicidas, en el rango de edad, en aparentes buenas condiciones de salud y sin hábitos psicosociales.

Para llevar a cabo el estudio se aplicaron las normas éticas correspondientes, se incluyó la carta de consentimiento informado, de acuerdo con lo establecido en los principios éticos para la investigación médica en seres humanos de la declaración de Helsinki y el instrumento aplicado tanto a los trabajadores expuestos como al grupo control fue una entrevista.

Las determinaciones laboratoriales realizadas fueron: niveles de colinesterasa plasmática; parámetros hematimétricos; actividad enzimática de las transaminasas, creatinina y urea.

Para el análisis molecular de la CYP3A4, previamente se efectuó una extracción de ADN genómico a partir de $200 \mu \mathrm{L}$ de sangre total empleando kit comercial (PureLink ${ }^{\mathrm{TM}}$ Genomic DNA Mini Kit, Invitrogen USA); posteriormente, para la amplificación se aplicó la técnica de PCR convencional, usando cebadores específicos para las regiones que flanquean los distintos exones descritos. ${ }^{20}$

Los cebadores para PCR fueron diseñados con una temperatura de fusión óptima de $60^{\circ} \mathrm{C}$, con una longitud entre 15-25 nucleótidos. Se seleccionaron cebadores para amplificar parte de la región up stream 5' del CYP3A4 y todos los exones flanqueados de ambas regiones de intrones CYP3A4 (exones: EXONA nt1-71, EXONB nt4004-4097, EXONC nt6009-6061, EXOND nt11502-11601, EXONEF nt13956-14069/14335-14423, EXONG nt1568915837, EXONH nt16932-17059, EXONI nt17744-17810, EXONJ nt20166-20326, EXONK nt21912-22138, EXONL nt23198-23360, EXONM nt25950-26502. La PCR se llevó a cabo en un volumen total de $25 \mu \mathrm{L}$, usando la siguiente mezcla de reacción: $12.5 \mu \mathrm{L}$ Platinum Hot Start PCR 2X Master Mix (Invitrogen, USA), $10 \mu \mathrm{M}$ de cada uno de los cebadores, de 100 a 200 ng de ADN genómico y agua libre de nucleasa.

La amplificación se realizó en un termociclador automático (Seeamp ${ }^{\mathrm{TM}}$, Seegene, Corea del Sur) con los siguientes parámetros: desnaturalización por 12 min a 93 ${ }^{\circ} \mathrm{C}$, seguida de 40 ciclos de 30 seg a $94{ }^{\circ} \mathrm{C}$, 30 seg de temperatura de hibridación variable, 2.30 min de extensión a $72{ }^{\circ} \mathrm{C}$ y una final por $10 \mathrm{~min}$ a $72{ }^{\circ} \mathrm{C}$. Los productos de PCR se analizaron por electroforesis en un gel de agarosa al $2 \%$ y se visualizaron en un Transluminador UVP ${ }^{\circledR}$.

Los eluidos de ADN de las muestras de pacientes y controles fueron trasladadas a través de Biotecnología Lambda desde Venezuela hasta la ciudad de Seúl en Corea del Sur (Laboratorio Macrogen), debidamente empacada y según los lineamientos indicados en las buenas prácticas de laboratorio para el procesamiento de secuenciación de ácidos nucleicos por secuenciación capilar de productos de PCR y análisis bioinformático de los resultados.

Cada muestra se usó como ADN templado para la reacción de amplificación por PCR para la obtención de productos fluorescentes marcados con la implementación de la química BigDye Terminator v 3.1 Cycle Sequencing. 
Posteriormente los productos de esta PCR fueron purificados mediante la implementación de columnas de afinidad para la obtención de un producto libre de potenciales interferentes del proceso de detección de fluorescencia.

Los productos de la PCR marcados con fluorescencia fueron analizados mediante tecnología de secuenciación por electroforesis capilar ABI 3730xI System, para la obtención de secuencias de nucleótidos entre 290-1,000 pb aproximadamente.

A cada secuencia se le realizó un análisis bioinformático básico (BlastN) mediante la plataforma del National Center for Biotechnologys Information, U.S. National Library of Medicine para conocer el gen de origen de la secuencia y porcentaje de identidad, mediante el alineamiento de la secuencia en estudio con las principales bases de datos a nivel mundial.

Finalmente, cada secuencia fue analizada individualmente mediante un alineamiento directo con la secuencia de referencia del gen CYP3A4 con el algoritmo de MUSCLE y análisis de electroferograma con el software Finch TV.

Durante la entrevista con las personas evaluadas del grupo expuesto realizada en su casa de habitación se hizo la lectura de las coordenadas de geolocalización mediante un equipo GPS Garmin ${ }^{\circledR}$ con señal detectada de al menos ocho satélites y reportada en grados decimales. Estas lecturas se utilizaron para el levantamiento del mapa bidimensional geográfico de los sujetos y sus valores de actividad de colinesterasa.

Todos los datos se analizaron a través del programa estadístico PAST versión 3.19. ${ }^{21}$ Se corroboró el ajuste de las variables cuantitativas a la distribución normal mediante la prueba de Kolmogórov-Smirnov, y se obtuvo la media aritmética y la desviación estándar. Las variables cualitativas se presentan en cuadros de distribución de frecuencias absolutas y relativas. Se calcularon medidas de asociación como el coeficiente de correlación de Pearson y se realizó la Prueba t-Student para comparar medias de dos muestras independientes (grupos expuesto y control). Se establecieron comparaciones de proporciones con la prueba Z . La diferencia entre los grupos se consideró significativa a un nivel de $p<0.05$. El mapa de geolocalización se realizó tomando los valores de latitud, longitud y actividad de colinesterasa como valores $x, y, z$ respectivamente $y$ aplicando el método de interpolación octal y suavizado por splines cúbicas para generar una matriz de 100 pixeles cuadrados en los ejes $x, y$. Los valores de actividad de colinesterasa (coordenada $z$ ) se convirtieron a una escala de pseudocolor con tono rojo como valor más alto y tono azul como el valor más bajo. Esta metodología permitió detectar en la geografía del área estudiada sectores de mayor o menor actividad de colinesterasa de las personas evaluadas.

\section{RESULTADOS}

En el presente estudio se evaluaron 30 individuos adultos de ambos sexos, aparentemente sanos, conformando dos grupos, uno expuesto (GE) y uno control (GC); el GE estuvo conformado por 20 trabajadores domiciliados en el sector Capachal de la Colonia Tovar del Estado Aragua ( $80 \%$ del sexo masculino y $20 \%$ femenino); y el GC por 10 trabajadores pertenecientes al personal administrativo de un Automercado de la Ciudad de Valencia, Estado Carabobo ( $90 \%$ del sexo masculino y $10 \%$ femenino), sin antecedentes de exposición a plaguicidas y en aparentes buenas condiciones de salud.

El GE presentó una edad promedio de $40.45 \pm 10.37$ años (rango: de 21 a 58 años) el GC, mostró una edad promedio $42.20 \pm 8.01$ años, rango de edad de 31 a 54 años y sin antecedentes de exposición a plaguicidas, sin diferencias estadísticamente significativas respecto al promedio de edad de ambos grupos $(p=0.644)$. La antigüedad en años de trabajo, en términos de promedio respecto al número total de trabajadores expuestos fue de $19.20 \pm 12.98$ años, con un límite inferior a dos años y un límite superior a 50 años.

Con relación a las actividades que ejecutan los trabajadores que componen el GE, 75.0\% mezclan y fumigan, sobre otras actividades asociadas $(Z=2.85 ; p=0.002)$.

Es importante resaltar que los 20 trabajadores expuestos manipulan directamente los plaguicidas y laboran aproximadamente $4.15 \pm 2.32$ horas diarias, con un valor promedio de antigüedad aplicando plaguicidas de $19.20 \pm 12.98$ años. Además, se pudo establecer que dichos trabajadores tienen un tiempo promedio por cada ciclo de fumigación de $6.20 \pm 2.04$ días al mes, realizando dichos ciclos de fumigación 12 veces en el año, lo que daría un estimado de 74 días de fumigación en un año calendario, siendo los de mayor frecuencia de uso Carbamato y Fungicida (Tabla 1).

En cuanto al nivel de instrucción de la muestra en estudio en el grupo de trabajadores expuestos, el mayor número culminó la primaria representando $60 \%$ y seis trabajadores son bachilleres, lo que corresponde a $30.0 \%$; por el contrario, en el grupo control, $60 \%$ son universitarios.

En la Tabla 2 se observan los promedios y desviación estándar de la actividad de la colinesterasa de los grupos evaluados. Para el GE se observó un valor promedio de 
la actividad de la colinesterasa de $6.4350 \pm 1.2465 \mathrm{U} / \mathrm{L}$ y para el GC un valor promedio de colinesterasa sérica de $8.2000 \pm 1.8749 \mathrm{U} / \mathrm{L}$. La media de la actividad de la colinesterasa sérica se encuentra dentro de los parámetros considerados normales (4.970-13.977 U/L) para ambos grupos según la técnica aplicada. Cabe destacar que a pesar de que los valores promedio de la colinesterasa en ambos grupos se ubican dentro de los parámetros norma-

\begin{tabular}{|c|c|c|}
\hline Insecticida/grado de toxicidad & $n^{*}$ & $\%$ \\
\hline Karate (fungicida/lb) & 11 & 55.0 \\
\hline Mercamil (carbamato/la) & 11 & 55.0 \\
\hline Bravo 500 (fungicida/lb) & 10 & 50.0 \\
\hline Folpán (fungicida/IV) & 7 & 35.0 \\
\hline Pyrine (OF/II) & 5 & 25.0 \\
\hline Curacrón (OF/II) & 5 & 25.0 \\
\hline Captán (fungicida/lb) & 4 & 20.0 \\
\hline Curazín (fungicida/III) & 4 & 20.0 \\
\hline Paratión (OF/la) & 3 & 15.0 \\
\hline Malatión (OF/III) & 3 & 15.0 \\
\hline Amidor (OF/III) & 3 & 15.0 \\
\hline Fulgor (carbamato/III) & 3 & 15.0 \\
\hline Gramonzón (herbicida/II) & 3 & 15.0 \\
\hline Manzate (fungicida/III) & 3 & 15.0 \\
\hline Danol (OF/la) & 2 & 10.0 \\
\hline Difós (OF/IV) & 2 & 10.0 \\
\hline Triazol (fungicida/II) & 1 & 5.0 \\
\hline Score (fungicida/III) & 1 & 5.0 \\
\hline Curacarb (fungicida/III) & 1 & 5.0 \\
\hline Fugitane (OF/IV) & 1 & 5.0 \\
\hline Kuik (carbamato/lb) & 1 & 5.0 \\
\hline Glifosán (herbicida/IV) & 1 & 5.0 \\
\hline Sumo (insecticida/IV) & 1 & 5.0 \\
\hline Clorfluoazuro (insecticida/IV) & 1 & 5.0 \\
\hline Drago (piretroide/II) & 1 & 5.0 \\
\hline \multicolumn{3}{|c|}{$\begin{array}{l}\text { OF }=\text { organofosforados. } \\
\text { * Número de trabajadores con respuesta afirmativa. } \\
\text { Fuente: Entrevista realizada. }\end{array}$} \\
\hline
\end{tabular}

les, $15.0 \%$ de los valores de colinesterasa obtenidos en el GE (tres trabajadores) se ubican por debajo del rango de normalidad. Adicionalmente, existe diferencia estadísticamente significativa $(p=0.005)$ entre las medias para el biomarcador de efecto en estudio, esto indica que la exposición a plaguicidas sí afecta los niveles de colinesterasa sérica en los agricultores expuestos a plaguicidas. Cuando se comparan las medias del valor de colinesterasa en el grupo expuesto según el sexo, no existe diferencia estadísticamente significativa ( $p=0.351$ ).

Según la información suministrada por el grupo estudiado $70 \%$ conoce los efectos nocivos para la salud causados por los plaguicidas, 55\% conoce el significado del rótulo de los envases y $5 \%$ los usa en su hogar (sólo un trabajador que refirió usar piretroide de forma ocasional). Respecto a quien suministró la información sobre el uso de plaguicidas, la mayoría refirió que fue suministrada por los compañeros de labores (55\%), 15\% por agrotécnico, $15 \%$ por un vecino y $15 \%$ a través de lectura del folleto.

En cuanto a las manifestaciones clínicas, los signos y síntomas referidos por los trabajadores expuestos fueron: $30 \%$ alergias y $30 \%$ picazón, con predominio estadísticamente significativo en comparación con los trabajadores del grupo no expuesto $(Z=2.21 ; p=0.01)$.

En cuanto a las medidas de protección empleadas por los trabajadores expuestos, $100 \%$ de ellos las utilizan. Cien por ciento de los trabajadores del grupo expuesto lava la ropa de trabajo aparte de la ropa normal, no ingiere alimentos en la zona de fumigación, se cambia la ropa después de la jornada, usa el uniforme lavado después de la fumigación, cambia los equipos de protección personal cuando están dañados, se lava las manos antes de comer y no se baña en ríos cercanos a su vivienda; $95 \%$ se lava las manos inmediatamente después de la jornada, 70\% toma agua durante la jornada, $70 \%$ se baña inmediatamente después de fumigar y $30 \%$ lo hace algunas horas después de fumigar.

Los datos suministrados por los trabajadores demuestran que $70 \%$ guarda los plaguicidas en un depósito aislado; $5 \%$ quema el envase usado sin lavado previo, $40 \%$ lo entierra con lavado previo y $40 \%$ lo quema y

Tabla 2: Actividad de la colinesterasa en el grupo expuesto a plaguicidas y grupo control.

\begin{tabular}{lccccc} 
Colinesterasa & $n$ & Media \pm DE & Mín. & Máx. & $p^{*}$ \\
\hline Grupo expuesto & 20 & $6.4350 \pm 1.2465$ & 4.20 & 8.50 & 12.40 \\
Grupo control & 10 & $8.2000 \pm 1.8749$ & 6.00 & 0.005 \\
\hline
\end{tabular}

DE = desviación estándar. * Prueba t de Student. Fuente: Entrevista realizada. 
entierra con lavado previo; $10 \%$ acumula los envases en un vertedero de basura, $5 \%$ lo acumula en el almacén y en el vertedero de basura.

Se detectaron diferencias estadísticamente significativas entre los grupos para los valores de hemoglobina, hematocrito, VCM, HCM, monocitos y triglicéridos al comparar las medias de los GE y GC (Tabla 3).

Se evidenció que existe correlación estadísticamente significativa ( $p<0.05)$, con la urea, de hecho, una correlación positiva media y estadísticamente significativa $(\mathrm{R}=0.500 ; \mathrm{p}=0.025)$ y una correlación positiva débil y estadísticamente significativa entre la colinesterasa y la TGP $(R=0.455 ; p=0.044)$.

En los 30 trabajadores evaluados la expresión genética de la CYP3A4 estuvo presente para el Exon A, Exon E y Exon $M$, para una prevalencia de $100 \%$ en ambos grupos. El Exón F se expresó en 83.3\% de la muestra, con diferencias estadísticamente significativas entre las prevalencias $(Z=2.94 ; p=0.001)$. El Exón K estuvo presente en $86.7 \%$ del total estudiado, sin diferencias estadísticamente significativas $(Z=0.19 ; p=0.42)$. El Exón $\mathrm{H}$ estuvo presente sólo en un trabajador expuesto (3.3\% de la muestra), sin diferencias significativas respecto a los no expuestos $(Z=0.36 ; p=0.35)$.

No se evidenciaron diferencias estadísticamente significativas al comparar las medias de acetilcolinesterasa en los grupos expuesto y no expuesto según la presencia o ausencia de los exones F, H y K ( $p>0.05)$, ni al comparar las medias de los parámetros hematimétricos y bioquímicos estudiados en los grupos expuesto y no expuesto según la presencia de los exones mencionados. Tampoco hubo correlaciones estadísticamente significativas entre

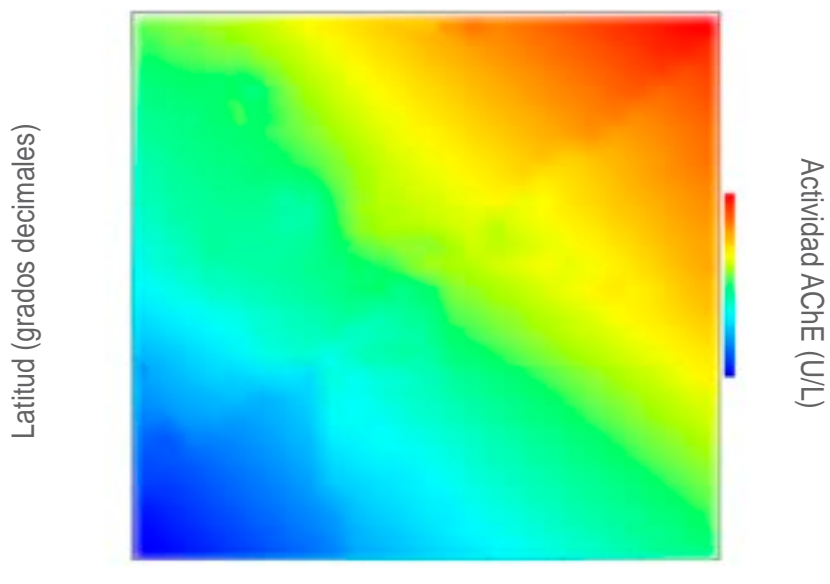

Longitud (grados decimales)

Figura 1: Localización geográfica de los expuestos ocupacionalmente a plaguicidas vs. actividad de la colinesterasa. Fuente: Exámenes de laboratorio realizados.

Tabla 3: Parámetros hematimétricos y bioquímicos de ambos grupos en estudio.

\begin{tabular}{|c|c|c|c|c|c|c|}
\hline \multirow{2}{*}{$\begin{array}{l}\text { Parámetros } \\
\text { hematimétricos y } \\
\text { bioquímicos }\end{array}$} & \multicolumn{2}{|c|}{ Grupo expuesto } & \multicolumn{2}{|c|}{ Grupo control } & \multirow[t]{2}{*}{ p } & \multirow{2}{*}{$\begin{array}{l}\text { Valores } \\
\text { de } \\
\text { referencia }\end{array}$} \\
\hline & $\mathrm{n}$ & Media $\pm \mathrm{DE}$ & $n$ & Media $\pm \mathrm{DE}$ & & \\
\hline Hemoglobina (g/dL) & 20 & $15.5 \pm 1.0973$ & 10 & $14.02 \pm 1.1669$ & 0.002 & $12.0-16.0$ \\
\hline Hematocrito (\%) & 20 & $46.95 \pm 3.9204$ & 10 & $42.5 \pm 2.9097$ & 0.004 & $40.0-50.0$ \\
\hline Hematíes $\left(\times 10^{6} / \mathrm{mm}^{3}\right)$ & 20 & $5.12 \pm 0.3319$ & 10 & $5.102 \pm 0.5016$ & 0.874 & $4.0-5.50$ \\
\hline Leucocitos $\left(\times 10^{3} / \mathrm{mm}^{3}\right)$ & 20 & $6.99 \pm 1.8123$ & 10 & $6.00 \pm 1.3646$ & 0.138 & $4.0-10.0$ \\
\hline $\operatorname{VCM}(f \mathrm{~L})$ & 20 & $91.62 \pm 4.9629$ & 10 & $83.83 \pm 7.5761$ & 0.020 & $82-95$ \\
\hline CHCM (g/dL) & 20 & $33.05 \pm 0.8204$ & 10 & $32.97 \pm 1.0499$ & 0.809 & $31.5-35.5$ \\
\hline $\mathrm{HCM}(\mathrm{pg})$ & 20 & $30.28 \pm 1.6897$ & 10 & $27.67 \pm 3.0313$ & 0.005 & $27-32$ \\
\hline Linfocitos (\%) & 20 & $33.05 \pm 6.2532$ & 10 & $35.90 \pm 4.8408$ & 0.218 & $17.0-48.0$ \\
\hline Monocitos (\%) & 20 & $3.20 \pm 2.0672$ & 10 & $6.10 \pm 2.6012$ & 0.002 & $0-8$ \\
\hline Eosinófilos (\%) & 20 & $2.90 \pm 2.2918$ & 10 & $2.70 \pm 1.0593$ & 0.746 & $0-6$ \\
\hline Neutrófilos (\%) & 20 & $59.85 \pm 7.4358$ & 10 & $55.3 \pm 5.4782$ & 0.098 & $43.0-76.0$ \\
\hline Plaquetas $\left(\times 10^{3} / \mathrm{mm}^{3}\right)$ & 20 & $220.95 \pm 36.5894$ & 10 & $235.8 \pm 38.9638$ & 0.314 & $150-450$ \\
\hline Urea (mg/dL) & 20 & $27.14 \pm 9.9538$ & 10 & $32.78 \pm 23.0632$ & 0.353 & $15.0-45.0$ \\
\hline Creatinina (mg/dL) & 20 & $0.75 \pm 0.1496$ & 10 & $0.86 \pm 0.1574$ & 0.076 & $0.5-1.4$ \\
\hline TGO/AST (U/L) & 20 & $28.80 \pm 9.0588$ & 10 & $31.00 \pm 8.3133$ & 0.525 & $0-40.0$ \\
\hline TGP/ALT (U/L) & 20 & $28.87 \pm 12.8328$ & 10 & $37.30 \pm 11.5185$ & 0.091 & $0-38.0$ \\
\hline
\end{tabular}

$\mathrm{DE}$ = desviación estándar; $\mathrm{VCM}=$ volumen corpuscular medio; $\mathrm{CHCM}=$ concentración de hemoglobina corpuscular media; TGO/AST = aspartato aminotransferasa; TGP/ALT = alanina aminotransferasa. Fuente: Entrevista realizada. 
CYP3A4 Exón F

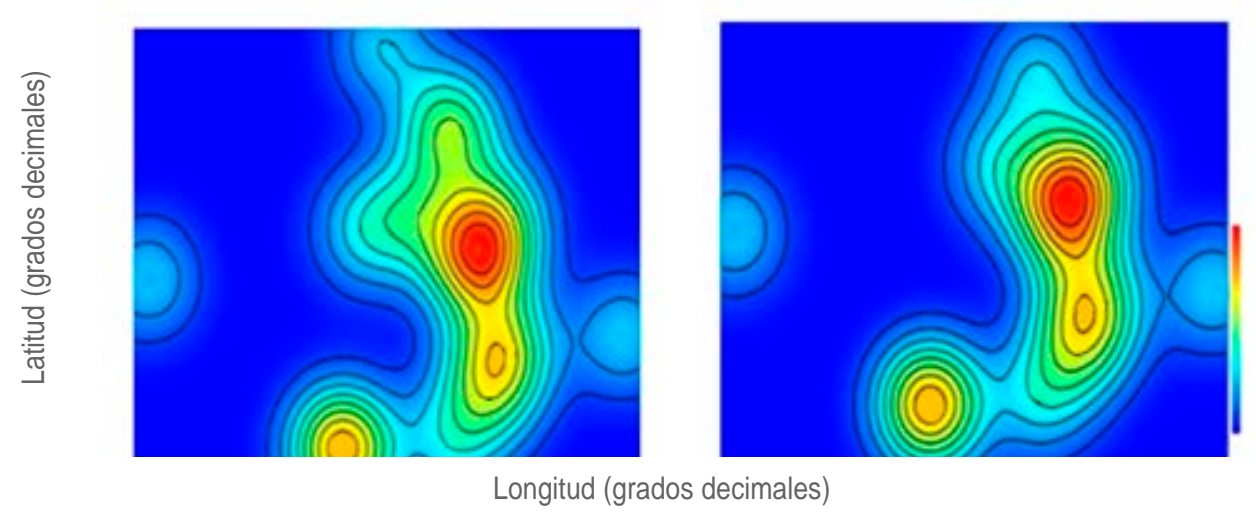

CYP3A4 Exón $\mathrm{K}$

Longitud (grados decimales)

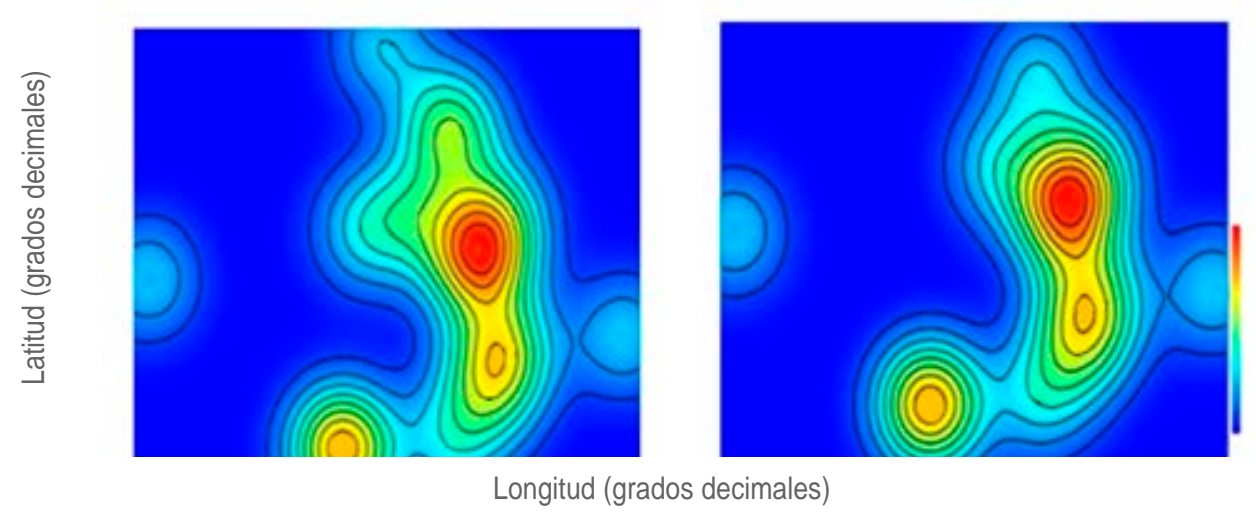

Localización geográfica de los expuestos ocupacionalmente a plaguicidas vs. expresión

CYP3A4.

Fuente: Exámenes de laboratorio realizados.

los valores de colinesterasa y la presencia de los exones $\mathrm{F}$, $\mathrm{H}$ y K. No se evidenciaron correlaciones estadísticamente significativas entre los valores de colinesterasa y la sintomatología ni entre la expresión de exones y la sintomatología, entre los trabajadores expuestos ocupacionalmente a los plaguicidas inhibidores de la colinesterasa.

El exón expresado con mayor frecuencia fue el $\mathrm{M}$, tanto en el grupo control (100\%) como en el grupo expuesto $(81.8 \%)$. El hallazgo más frecuente en el grupo control fue la variante alélica intrónica $A>C$ 30,834 (heterocigoto) $75 \%$ y en el grupo expuesto fue la variante alélica UTR T>A 1,787 (heterocigoto) 36.4\%, sin diferencias estadísticamente significativas $(p>0.05)$.

La localización geográfica del estudio en los pacientes con baja actividad de colinesterasa fue al suroeste del caserío agrícola Capachal de Palmarito, lo cual sugiere que esta área tiene una actividad agrícola intensa y por ende mayor uso de plaguicidas inhibidores de la colinesterasa, en comparación con las restantes áreas en el mapa (Figura 1).

En los mapas de geolocalización de densidad de pacientes expuestos a plaguicidas con expresión del exón F (izquierda) o K (derecha) en el área estudiada, la escala de pseudocolores indica la mayor (roja) o la menor (azul) densidad relativa (unidades arbitrarias) de pacientes con expresión de cada exón (Figura 2).

\section{DISCUSIÓN}

En el presente estudio se evaluaron 30 individuos adultos de ambos sexos, aparentemente sanos, conformando dos grupos, uno expuesto (GE) y uno control (GC); una situación similar en la media del grupo expuesto fue presentada por Cárdenas y colaboradores (2010) en su estudio realizado en Colombia, del total de trabajadores participantes $78.9 \%$ eran hombres y $21.1 \%$ mujeres con rango de edad entre 18 y 40 años en ambos sexos expuestos a plaguicidas. ${ }^{22}$ En 2015 se llevó a cabo otro estudio con una edad media para individuos del grupo expuesto de $39.6 \pm 10.8$, similar a los valores reportados por este estudio. ${ }^{23}$

La antigüedad en años de trabajo de los pacientes expuestos con límite inferior a dos años hasta un límite superior a 50 años, promedio de horas diarias de trabajo de $4.15 \pm 2.32$ y un valor promedio de antigüedad aplicando plaguicidas de $19.20 \pm 12.98$, años ejecutando actividades de mezcla y fumigación en $75 \%$ de los encuestados y un promedio de tiempo de fumigación de $6.20 \pm 2.04$ días al mes. Estos datos coinciden con los resultados encontrados por Gómez y su equipo (2010) ${ }^{24}$ y por Toro-Osorio y colegas (2017). ${ }^{25}$

En relación al estudio, $60 \%$ de los encuestados tienen un nivel de primaria completo y $30 \%$ son bachilleres. Por el contrario, el estudio de Castiblanco en 2014, 72.1\% ha alcanzado la secundaria y tan sólo $5 \%$ es profesional y cuenta con maestría. ${ }^{26}$

El valor de colinesterasa de los grupos evaluados fue en promedio para el grupo expuesto de $6.4350 \pm$ $1.2465 \mathrm{U} / \mathrm{L}$ y para el grupo control un valor promedio de $8.2000 \pm 1.8749 \mathrm{U} / \mathrm{L}$, detectándose diferencia estadísticamente significativa $(p<0.05)$ entre las medias para el biomarcador de efecto en estudio. Similares resultados se obtuvieron de los estudios de Castiblanco (2014) y Matheus y colaboradores (2017), reportándose diferencias significativas con una $p<0.01$ y $p<0.000$ respectivamente, ${ }^{26,27}$ lo que permite inferir que al mantenerse condiciones hostiles o de estrés en los individuos se esté dando una estimulación del sistema metabólico como una primera respuesta adaptativa al ambiente; esta respuesta adaptativa fue descrita por primera vez por Kashyap en 1984 y luego fue nuevamente reportada por García y su equipo (2015), quienes establecieron que ante una exposición crónica a plaguicidas, el sistema 
metabólico responde con un incremento de la actividad enzimática con el fin de establecer nuevamente el equilibrio del sistema; ${ }^{27,28}$ en cambio en el estudio de Toro-Osorio y colegas (2017) no existe diferencia significativa en los niveles de exposición a plaguicidas entre los agricultores estudiados. ${ }^{25}$

Los plaguicidas más utilizados por los trabajadores de este estudio son del tipo organofosforados y carbamatos; dentro del grupo de los organofosforados predominan: 25\% Pyrine, 25\% Curacrón, 15\% Paratión, 10\% Danol, de categorías II y la (moderadamente tóxico y extremadamente tóxico respectivamente), y en el caso de los Carbamatos los más empleados fueron: 55\% Mercamil, 15\% Fulgor, 5\% Kuik, de categoría la, III y lb, extremadamente, moderadamente y altamente tóxico, respectivamente. Estos datos tienen concordancia con los aportados por estudios de Gómez y colaboradores (2010) y Toro y su equipo (2017), los organofosforados son los insecticidas más utilizados para el control de plagas en los cultivos, tanto a nivel internacional como en Venezuela, éstos junto con los carbamatos son los frecuentes involucrados en intoxicaciones en todo el mundo. ${ }^{24,25}$ Sin embargo, según la información suministrada por el grupo estudiado de Capachal de Palmarito, 70\% conoce los efectos nocivos para la salud causada por los plaguicidas que utilizan.

Para este estudio, 95\% de los trabajadores recibió instrucciones para el manejo de los plaguicidas, 55\% conoce el significado del rótulo de los envases en los que se expende el plaguicida y $5 \%$ usa plaguicidas en su hogar. Respecto a quién suministró la información sobre el uso de plaguicidas, la mayoría refirió que fue suministrada por los compañeros de labores (55\%), 15\% por agrotécnico, 15\% por un vecino y $15 \%$ a través de lectura del folleto, situación similar a la presentada por Duran y colegas (2017), quienes reportan un porcentaje significativo de agricultores que emplean plaguicidas sin ninguna capacitación formal previa, lo cual representa un enorme riesgo, ya que es un tema muy amplio y de alta complejidad. ${ }^{29}$

Los trabajadores agrícolas, la mayoría de bajo nivel sociocultural, no utilizan las debidas medidas de protección, ya sea por falta de conocimiento, descuido o porque las empresas no proveen del equipamiento necesario a sus trabajadores, planteamientos que hacen considerar, junto a otros, a la agricultura como una de las actividades más peligrosas, según la OIT, ya que genera trabajo físico excesivo, estrés psicológico, desequilibrios en el ritmo del trabajo y por consiguiente mayores riesgos de accidentes y enfermedades por exposición sin control al uso excesivo de plaguicidas, sin periodos de carencia, lo que se traduce como explotación laboral, considerada por la OIT como la nueva forma de esclavitud del siglo XXI. ${ }^{30}$
Siguiendo con el punto relacionado con el manejo adecuado de la información para el uso correcto de plaguicidas, en este estudio sólo $70 \%$ de los trabajadores toman agua durante la jornada, $30 \%$ lo hace horas después de culminar la fumigación, resultados comparables con el estudio de Toro y colaboradores (2017), ${ }^{25}$ al igual que los reportados por Cárdenas y su equipo. ${ }^{22} \mathrm{El}$ incumplimiento de estas medidas higiénicas propician la presencia de síntomas asociados con la exposición a plaguicidas, mezclas de solventes orgánicos y metales pesados, por lo cual resulta interesante plantear la aplicación de una batería de test neuroconductuales para detectar precozmente los posibles efectos neucomportamentales de los plaguicidas en los sujetos estudiados. ${ }^{31,32}$ El estudio llevado a cabo por Muñoz (2011) refirió la prevalencia en toda la muestra en estudio del síndrome muscarínico. ${ }^{33}$

Los datos suministrados por los trabajadores demuestran que $70 \%$ guarda los plaguicidas en un depósito aislado, $40 \%$ de los agricultores del estudio queman y entierran el envase usado con lavado previo y $40 \%$ sólo lo entierra sin tratamiento; similares porcentajes refleja el trabajo de Varona y colegas (2016); en cambio García y colaboradores (2006) refieren que la mayoría del grupo expuesto almacena correctamente los plaguicidas. ${ }^{30,34}$

Respecto a los resultados de los exámenes de laboratorio practicados, todos los parámetros se encuentran dentro de los valores normales. Se detectan diferencias estadísticamente significativas entre los grupos, los valores de hemoglobina, hematocrito, VCM y HCM y monocitos pueden justificarse por la diferencia de altura con respecto al lugar de residencia tanto del grupo expuesto como del grupo control. Sin embargo, en estudios realizados por Ahmadi y su equipo (2018), los resultados fueron contrarios a los expuestos en este estudio, pudiendo asociar estos hallazgos con daños oxidativos, y presencia de citoquinas inflamatorias inducidas por la presencia de xenobióticos. ${ }^{35}$

A nivel renal, con la urea se evidenció correlación estadísticamente significativa, analito de suma utilidad a los fines de determinar una alteración en la función renal debida a una disminución en la capacidad del organismo para eliminar sustancias químicas potencialmente genotóxicas, incrementando por tanto el riesgo de daño genético y enfermedades asociadas al mismo; sin embargo, estudios llevados a cabo por Lebov y colegas (2016) indican que se requieren de estudios epidemiológicos adicionales para confirmar el efecto nefrotóxico y desarrollo de posteriores patologías renales por exposición. ${ }^{36}$

Es importante resaltar que la diferencia significativa entre los dos grupos para los valores de TGP pueden estar relacionados al hecho de que los plaguicidas se metabo- 
lizan en el hígado y dicha enzima es específica en este órgano, lo que facilita la identificación de hepatopatías y de otros padecimientos. En este contexto, nuestros resultados concuerdan con lo reportado por otros autores, los plaguicidas organofosforados y carbamatos dañan al hígado durante las intoxicaciones subaguda y crónica, debido a la generación de estrés oxidante caracterizado por lipoperoxidación membranal de los hepatocitos, disminución de la síntesis de ATP, daño estructural y necrosis celular, incremento en el depósito de lípidos y disminución de glucógeno. ${ }^{37}$

La metodología de geolocalización descrita en el presente estudio permitió detectar el predominio de sujetos con baja actividad colinesterásica en el cuadrante suroeste del caserío agrícola Capachal de Palmarito, lo cual sugiere que esta área tiene una actividad agrícola intensa y coincide con su residencia, por ende, mayor uso de plaguicidas inhibidores de la colinesterasa en comparación con las restantes áreas en el mapa.

Diversos estudios sugieren que las diferencias en la expresión de la CYP constituyen una de las principales fuentes de variabilidad interindividual en la respuesta a xenobióticos. En este contexto, el presente estudio puso en evidencia la expresión del Exón F de la CYP3A4 en 100\% de los expuestos a plaguicidas a diferencia del $50 \%$ de expresión del mismo gen en los no expuestos y la expresión del Exón $\mathrm{H}$ de la prenombrada isoforma en un paciente expuesto ocupacionalmente para la metabolización del mismo xenobiótico. Por lo expuesto anteriormente, diferencias en la regulación, expresión y actividades de las enzimas de la CYP en fase I y II del metabolismo pueden ser factores para definir la susceptibilidad del individuo y podrían contribuir a los efectos en la salud en el caso de exposiciones a plaguicidas a largo plazo, lo cual debería tenerse en cuenta en futuros estudios a fin de mejorar la predicción de tales efectos; dichos resultados coinciden con los reportados por otros investigadores. ${ }^{38}$

Los resultados del estudio de geolocalización para la expresión de los exones de la isoforma CYP3A4 sugieren una densidad aumentada de pacientes expuestos con expresión de exones $\mathrm{F}$ y $\mathrm{K}$ incrementada en el sector nororiental del área estudiada con diferencias en las densidades para cada uno de estos exones. Estos resultados de densidad podrían ser el resultado de una mayor exposición a plaguicidas en estas áreas, a una mayor agrupación de estos pacientes o a una combinación de ambos factores, pero en cualquiera de estas situaciones requiere evaluar la posibilidad de reubicación de los sitios de vivienda.

Las variantes alélicas encontradas para CYP3A4 en la presente investigación fueron consultadas en las bases de datos ClinVar y Genebank, 16,39 dichas variantes no están relacionadas con la exposición o efecto del xenobiótico en el genotipo de los individuos estudiados, ${ }^{39}$ la razón puede deberse a una función en forma concertada de la CYP3A4 con la glicoproteína P (Gp-P) para reducir la concentración intracelular de xenobióticos; la Gp-P proteína transmembrana que contribuye a la disminución de la absorción y un aumento de la eliminación del xenobiótico. Es por ello que la expresión conjunta del CYP3A4 y de la Gp-P puede tener un papel sinérgico de protección y un papel importante en la modulación de la expresión de la CYP3A4; ${ }^{40}$ sin embargo, según investigaciones recientes, se requiere una caracterización más amplia y sistemática de esta relación, tomando en cuenta los posibles polimorfismos del transportador, los efectos de mezclas de plaguicidas y los llamados factores fisiológicos del individuo expuesto. ${ }^{41}$

\section{REFERENCIAS}

1. Torres D, Capote T. Agroquímicos un problema ambiental global: uso del análisis químico como herramienta para el monitoreo ambiental. Ecosistemas. 2004; 13 (3): 2-6.

2. Morell I, Hernández F. El agua en Castellón. Un reto para el siglo XXI. España: Universitat Jaime I; 2000. ISBN 84-8021-333-7.

3. Correa A. Manual de registro de plaguicidas para Centroamérica [Libro en línea]. FAO; 2011. [Consulta 06 de noviembre de 2016] Disponible en: http://www.fao.org/docrep/019/as399s/as399s.pdf

4. FAO. Bonn: La FAO lanza una guía para adaptar la agricultura al cambio climático. 2017. Disponible en: https://news.un.org/es/ story/2017/11/1421982 $\cdot$ Wg38vtLiblU.

5. FAO. Boletín informativo. 2018. Disponible en: http://www. fao.org/climatechange/epic/que-hacemos/que-es-la-agriculturaclimaticamente-inteligentemente/es/.Wqp9PujOUdV.

6. FAO. Boletín informativo 2019. [Consulta 08 de noviembre de 2019] Disponible en: http://www.fao.org/news/story/es/ item/180993/icode/

7. FAO y OMS. Las nuevas directrices sobre plaguicidas pretenden suprimir más rápidamente las toxinas peligrosas. Roma: 2016. Disponible en: http://www.fao.org/news/story/es/item/414021/icode/

8. Androutsopoulos V, Kanavouras K, Tsatsakis K. Role of paraoxonase 1 (PON1) in organophosphate metabolism: Implications in neurodegenerative diseases. Toxicol Appl Pharmacol. 2011; 256: 418-424.

9. PNUMA. Los Convenios sobre productos químicos y desechos peligrosos. 2004. Disponible en: http://archive.basel.int/pub/ threeConventions-s.pdf

10. Marrero S, González S, Guevara H, Eblen A. Evaluación de la exposición a organofosforados y carbamato en trabajadores de una comunidad agraria. Comunidad y Salud. 2017; 15 (1): 30-41.

11. FAO. Agricultura mundial hacia los años 2015/2030, estudio de la FAO. Roma, Italia: 2010. Disponible en: http://www.fao.org/ docrep/004/y3557s/y3557s00.htm

12. Programa de Seguridad y Salud en el Trabajo y Medio Ambiente. Departamento de la Protección del Trabajo. Oficina Internacional del Trabajo OIT. Seguridad y salud en la agricultura. Ginebra, Suiza: 2000. Disponible en: http://www.ilo.org/wcmsp5/groups/ public/@ed_protect/@protrav/@safework/documents/publication/ wcms_117460.pdf 
13. Gil M. Proyecto Internacional de Eliminación de los COP. Informe Ciudadano de la Situación de los Contaminantes Orgánicos Persistentes en Venezuela. Fundación Aguaclara; 2006. Disponible en: http://docplayer.es/15000477-Informe-ciudadano-de-lasituacion-de-los-contaminantes-organicos-persistentes-envenezuela.html

14. Comisión Venezolana de Normas Industriales (Ministerio de Fomento). Norma Venezolana. Caracas: FONDONORMA; 2002. COVENIN: 2709 .

15. Ministerio del Poder Popular para el Ambiente (MINAMB) y la Organización de Naciones Unidas para el Desarrollo Industrial (ONUDI). Plan Nacional de implantación del Convenio de Estocolmo sobre los COP. Caracas-Venezuela: 2013.

16. Gil M. Red de Acción en Plaguicidas y sus Alternativas en México y Latinoamérica. Proyecto Internacional de Eliminación de los COP. Informe Ciudadano de la Situación de los Contaminantes Orgánicos Persistentes en Venezuela. Caracas: 2006.

17. Repetto M, Repetto G. Toxicología fundamental. 4a edición. Ediciones Díaz Santos; 2009.

18. Buratti F, Testai E. Evidences for CYP3A4 autoactivation in the desulfuration of dimethoate by human liver. Toxicology. 2007; 241 (1-2): 33-46.

19. Fleitas L, Durán N, Miranda C, Lee K, Quiñones L. Estudio de polimorfismos genéticos en CYP3A4 y CYP2D6, y su papel en la susceptibilidad a cáncer de mama. Rev Hosp Clin Univ Chile. 2013; 24: 95-104.

20. Berno G, Zaccarelli M, Gori C, Tempestilli M, Antinori A, Perno $\mathrm{C}$ et al. Analysis of single-nucleotide polymorphisms (SNPs) in human CYP3A4 and CYP3A5 genes: potential implications for the metabolism of HIV drugs. BMC Medical Genetics. 2014; 15: 76 .

21. Hammer $\varnothing$, Harper DAT, Ryan PD. PAST: paleontological statistics software package for education and data analysis. Palaeontologia Electronica. 2001; 4 (1): 9. Disponible en: http://palaeo-electronica. org/2001_1/past/issue1_01.htm

22. Cardenas O, Silva E, Morales L, Ortiz J. Uso de plaguicidas inhibidores de inhibidores de acetilcolinesterasa en once entidades territoriales de salud en Colombia, 2002-2005. Biomédica. 2010; 30 (1): 95-106.

23. Rosales J. Uso de marcadores genotoxicológicos para la evaluación de agricultores expuestos a plaguicidas organofosforados. An Fac Med. 2015; 76 (3): 247-252.

24. Gómez M, Cáceres J. Toxicidad por insecticidas organofosforados en fumigadores de Campaña contra el Dengue, estado Aragua, Venezuela 2008. Bol Mal Salud Amb. 2010; 50 (1): 119-125. Disponible en: http://www.scielo.org.ve/pdf/bmsa/v50n1/art12.pdf

25. Toro B, Rojas A, Díaz J. Niveles de colinesterasa sérica en caficultores del Departamento de Caldas, Colombia. Rev Salud Publica. 2017; 19 (3): 318-324.

26. Castiblanco A. Caracterización de la actividad enzimática y polimorfismo genéticos de butirilcolinestrasa (BCHE) en una población de trabajadores expuestos a plaguicidas inhibidores de colinesterasa en el municipio de Soacha 2014 [Tesis Magister en Toxicología]. Bogotá, Colombia: Universidad Nacional de Colombia. Instituto de Biotecnología. Departamento de Toxicología. Facultad de Medicina; 2014.
27. Matheus T, Aular Y, Bolaños A, Fernández Y, Barrios E, Hung M. Actividad de butirilcolinesterasa y micronúcleos en trabajadores agrícolas expuestos a mezcla de plaguicidas. Salud de los Trabajadores. 2017; 25 (1): 23-26.

28. García R, Parrón T, Requena M, Alarcón R, Tsatsakis A, Hernández A. Occupational pesticide exposure and adverse health effects at the clinical, hematological and biochemical level. Life Sciences. 2015; 145: 274-283.

29. Duran A, González M, Vargas G, Mora D. Situaciones de riesgo potencial relacionadas con la aplicación de agroquímicos en los sistemas hortícolas. Agronomía Costarricense. 2017; 41 (2): 67-77.

30. Organización Internacional del Trabajo (OIT). Los Convenios de la OIT sobre seguridad y salud en el trabajo: una oportunidad para mejorar las condiciones y el medio ambiente de trabajo. Publicaciones, Centro Internacional de Formación de la OIT 2009. Disponible en: http://www.ilo.org/buenosaires/publicaciones/ WCMS_BAI_PUB_118/lang--es/index.htm

31. Otero G, Porcayo R, Aguirre D, Pedraza M. Estudios neuroconductuales en sujetos laboralmente expuestos a plaguicidas. Rev Int Contaminación Ambiental. 2000; 6 (2): 67-74.

32. Caraballo M, Blanco G. Evaluación neuropsicológica de trabajadores expuestos a solventes orgánicos en una empresa de transporte público. Revista Facultad de Medicina. 2005; 28 (1): 79-88.

33. Muñoz M. Aspectos bioéticos en el control y aplicación de plaguicidas en Chile. Acta Boeth [Internet]. 2011; 17 (1): 95104. Disponible en: www.revistas.uchile.cl/index.php/AB/article/ download/15683/16154

34. Varona M, Díaz S, Briceño L, Sánchez C, Torres C, Palma R et al. Determinantes sociales de la intoxicación por plaguicidas entre cultivadores de arroz en Colombia. Rev Salud Publica. 2016; 18 (4): 617-629.

35. Ahmadi N, Mandegary A, Jamshidzadeh A, Mohammadi M, Mohammadi M, Salari E et al. Hematological abnormality, oxidative Stress and genotoxicity induction in the Greenhouse Pesticide Sprayers. Toxics. 2018; 6 (1): 1-15.

36. Lebov J, Engel L, Richardson D, Hogan S, Hoppin J, Sadler D. Pesticide use and risk of en-stagerenal disease among licensed pesticide applicators in the agricultural health study. Occup Environ Med. 2016; 73 (1): 3-12.

37. Fuentes V, Quezada C, Martínez M, Jaramillo F, Rodríguez L, Jaramillo $\mathrm{F}$ et al. Hepatotoxicidad subaguda y crónica producida por el plaguicida paratión-metílico en la rata. Rev Mex Cienc Farm. 2011; 42 (3): 50-59.

38. Piperakis S, Petrakou E, Tsilimigakis S, Sagnou M, Monogiudis E, Haniotakis $\mathrm{G}$ et al. Biomonitoring with the comet assay of Greek greenhouse workers exposed to pesticides. Environ Mol Mutagen. 2003; 41 (2): 104-110.

39. National Center for Biotechnology Information (NCBI). U.S. National Library of Medicine; 2016. [Consulta 26 de noviembre de 2019] Disponible en: https://www.ncbi.nlm.nih.gov/pubmed/

40. Peralta G, Sánchez M, Echeverria S, Valdizan E, Armijo J. Glucoproteína $\mathrm{P}$ e infección por el virus de la inmunodeficiencia humana. Enferm Infecc Microbiol Clin. 2008; 26 (3): 150-159.

41. Guéniche N, Bruyere A, Le Vée M, Fardel O. Implication of human drug transporters to toxicokinetics and toxicity of pesticides. Pest Manag Sci. 2019; 76 (1): 18-25. 